\title{
Distributions, transports and fates of short- and medium-chain chlorinated paraffins in a typical river-estuary system
}

\author{
Xiaohui Pan a , Xiaomei Zhen ${ }^{\mathrm{a}, \mathrm{b}, \mathrm{c}}$, Chongguo Tian ${ }^{\mathrm{a}}$, Jianhui Tang ${ }^{\mathrm{a}, *}$ \\ a Key Laboratory of Coastal Environmental Processes and Ecological Remediation, Yantai Institute of Coastal Zone Research, Chinese Academy of Sciences, Yantai 264003, China \\ b State Key Laboratory of Organic Geochemistry, Guangzhou Institute of Geochemistry, Chinese Academy of Sciences, China \\ ${ }^{c}$ University of Chinese Academy of Sciences, Beijing 100049, China
}

\section{H I G H L I G H T S}

- MCPPs and SCCPs were widely detected in water and sediment of Xiaoqing River.

- CPs in Xiaoqing River were among the highest worldwide.

- SCCPs were the dominant species in the dissolved phase of river water.

- Cities were the most important sources of CPs.

- ETM detains long-chained and more chlorinated CPs into sediment.

\section{A R T I C L E I N F O}

\section{Article history:}

Received 5 July 2020

Received in revised form 16 August 2020

Accepted 16 August 2020

Available online 18 August 2020

Editor: Shuzhen Zhang

\section{Keywords:}

SCCPs

MCCP

Xiaoqing River

Estuarine turbidity maximum zone

Laizhou Bay

Source

Fate
G R A P H I C A L A B S T R A C T
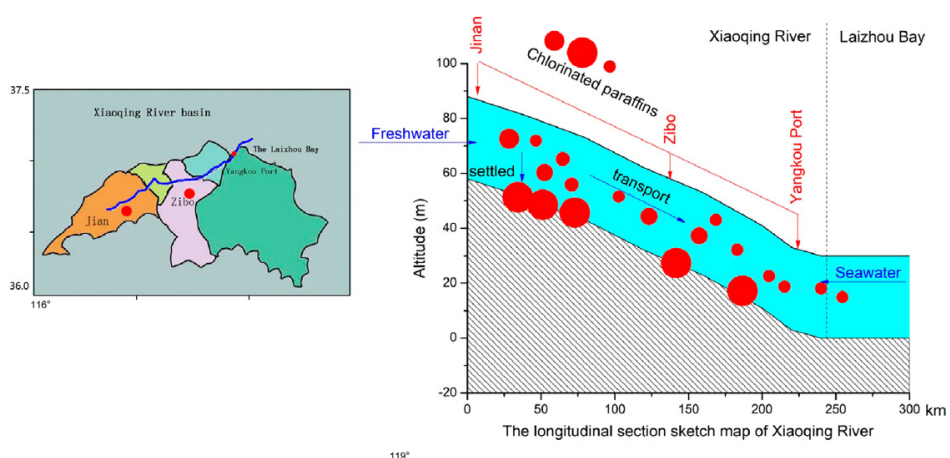

\section{A B S T R A C T}

Chlorinated paraffins (CPs) are widely employed in a variety of domestic and industrial products, and are ubiquitously detected in the environment. Short-chain chlorinated paraffins (SCCPs) have been listed in the Stockholm Convention as persistent organic pollutants (POPs), but not medium-chain chlorinated paraffins (MCCPs), even though they exhibit physicochemical properties and environmental behaviors similar to SCCPs. However, very limited data are available regarding their environmental behaviors and fates in river-estuary systems. China is the major producer of chlorinated paraffins (CPs), and Shandong Province is the main producer of CPs in China. Here, we investigated the distribution, transport, and fate of SCCPs and MCCPs in a heavily polluted river in Shandong Province, aiming to explore the distributions of CPs between dissolved and particulate phases, and between water and sediment phases, as well as the transport of CPs from river headwaters to estuaries and the roles of the estuarine turbidity maximum zone (ETM) on the fate of CPs. $\mathrm{CP}$ concentrations in sediments were 9.1-16,000 ng/g dw (mean value: $1000 \mathrm{ng} / \mathrm{g} \mathrm{dw}$ ) for SCCPs and 2.4-27,000 ng/g dw (mean value: $4400 \mathrm{ng} / \mathrm{g} \mathrm{dw}$ ) for MCCPs. In the water column, CP concentrations were 7.4-470 ng/L for SCCPs (mean value: $43 \mathrm{ng} / \mathrm{L}$ ) and 4.0-120 ng/L for MCCPs (mean value: $27 \mathrm{ng} / \mathrm{L}$ ). CP concentrations in riverine sediments were among the highest worldwide. SCCPs accounted for 95\% of CPs (sum of SCCPs and MCCPs) in the dissolved phase. Cities around the river basin were found to be important pollution sources for CPs. Long-chained and more chlorinated congeners with larger LogKow values might be more likely to be 'salted-out', and thus, will be sequestrated in sediments in the ETM, while those lighter congener groups with relatively high water solubility were prone to be transported by water flow to larger distances.

(c) 2020 Elsevier B.V. All rights reserved.

\footnotetext{
* Corresponding author.

E-mail address: jhtang@yic.ac.cn (J. Tang).
} 


\section{Introduction}

Chlorinated paraffins (CPs) are among the most complex halogenated contaminants, featuring large variations in carbon chain lengths $\left(\mathrm{C}_{10}-\mathrm{C}_{37}\right)$ and chlorination degrees $(40-70 \mathrm{~m} / \mathrm{m} \% \mathrm{Cl}$ ) (Brits et al., 2020). Based on their chain lengths, CPs can be subdivided into short-chain (SCCPs, $\mathrm{C}_{10}-\mathrm{C}_{13}$ ), medium-chain (MCCPs, $\mathrm{C}_{14}-\mathrm{C}_{17}$ ), and long-chain (LCCPs, $\mathrm{C}_{18}-\mathrm{C}_{37}$ ) paraffins. CPs have been widely used as lubricants, plasticizers, and additives in a great variety of industrial and consumer products due to their broad chemical properties. As a result, they have been widely detected in various environmental compartments (Bayen et al., 2006; Wei et al., 2016). A large number of industrial $\mathrm{CP}$ applications have attracted scientific concern and regulatory scrutiny of SCCPs. Numerous literature shows that SCCPs match the definition of persistent organic pollutants (UNEP, 2016). In May 2017, the POPs review committee of the Stockholm Convention (SC) decided to list the SCCPs in Annex A as a group of new POPs at its eighth meeting. Owing to the ban on SCCPs, a shift to MCCPs in manufacturing and consumption has been underway, resulting in widespread contamination of wildlife (Du et al., 2018). MCCPs have also been detected in foodstuff as well as indoor dust, and pose a potential threat to human health (Glüge et al., 2018; Huang et al., 2018; Sprengel et al., 2019). However, even though their chemical structures, environmental behaviors, and toxicities were similar to those of SCCPs, the production and usage of MCCPs have not been restricted (Glüge et al., 2018).

China has emerged as the largest producer and consumer of CPs worldwide. Annual production volumes have increased rapidly from 515,000 tons in 2007 to $1,124,000$ tons in 2013 and are estimated to grow at an annual rate of $10.9 \%$ or higher in the future (Zhang et al., 2017). However, no rigid measures have been taken in the CP commercial market in China until now. Shandong Province is one of the largest producers of CPs in China, which also results in largest emissions into the air and water (Jiang et al., 2017; Zhang et al., 2017). Laizhou Bay is a semi-enclosed bay surrounded by the Shandong Province, extending from Qimu Cape to the Yellow River Estuary, with a length of $320 \mathrm{~km}$ on the coastline. It is severely polluted by anthropogenic pollutants discharged from more than fifteen rivers and numerous wastewater pipelines. Our previous studies have reported the pollution by several toxic substances in the rivers and in the marine environment of the Laizhou Bay area (Pan et al., 2011; Zhang et al., 2012; Zhao et al., 2013; Zhong et al., 2011).

Among all the rivers flowing into Laizhou Bay, the artificial Xiaoqing River is a large contributor to pollution fluxes and runoff (Pan et al., 2011; Pan et al., 2018; Zhong et al., 2011). It originates from Jinan City and flows downstream through the heavily urbanized and industrialized cities of Zibo, Weifang, Binzhou, Dongying, and finally discharges into Laizhou Bay. Our previous study has revealed the heavily contamination by halogenated flame-retardants (HRFs) and perfluoroalkyl substances (PFASs) in the Xiaoqing River and the likely threats of riverine input to Laizhou Bay (Heydebreck et al., 2015; Zhen et al., 2018). The present study focused on the distribution and transportation of CPs from the Xiaoqing River to the marine environment. Both water and sediment samples were collected and analyzed to uncover the occurrence and fate of $\mathrm{CPs}$ from the Xiaoqing River in relation to the Laizhou Bay environment. From this analysis, we expected to determine the influence of human activities along a river basin on the water quality and further to obtain useful information about the control over emissions of CPs to ecosystems that would be useful for the protection of human health.

\section{Materials and methods}

\subsection{Sample preparation and analysis}

Twenty-two sediment samples and 30 water samples were obtained from the upper reaches to the estuary of the Xiaoqing River in April
2014 (Fig. 1). Freeze-dried sediment samples (40 g) and filters (from 20 to $40 \mathrm{~L}$ water per sample) containing suspended CPs were spiked with $10 \mathrm{ng}$ of surrogate standard $\left(\left[{ }^{13} \mathrm{C}_{10}\right]\right.$ trans-chlordane, purchased from Cambridge Isotope Laboratories) and then extracted by dichloromethane (DCM) by Soxhlet method for $24 \mathrm{~h}$. The dissolved phase (20-40 L water) CPs were allowed to pass through an Amberlite ${ }^{\circledR}$ XAD-2 resin column $(30 \times 4 \mathrm{~cm}$ i.d $)$ and then extracted in a modified Soxhlet apparatus for $24 \mathrm{~h}$ using DCM. Elemental sulfur was removed by adding activated copper granules into the extraction flasks. All the extracts were further concentrated and subjected to a cleanup procedure. The eluent was concentrated to $\sim 100 \mu \mathrm{L}$ in isooctane and spiked with $20 \mathrm{ng}$ of $\left[{ }^{13} \mathrm{C}_{8}\right]$ mirex (as an internal standard) before injected. More details about the sample pretreatment are presented in the supplementary information (SI). Detailed sampling information has been described in our previous study (Zhen et al., 2018).

A gas chromatograph coupled with an electron capture negative ion - mass spectrometer (GC/NCI-MS, Agilent 7890-5975C, USA) was used to monitor the two most abundant isotopes of the $[\mathrm{M}-\mathrm{Cl}]^{-}$ions of SCCPs and MCCPs with 5-10 chlorine atoms (Iozza et al., 2009; Reth and Oehme, 2004). Three SCCPs $(51.5 \%, 55.5 \%$, and $63.0 \% \mathrm{Cl})$, three MCCP references $(42.0 \%, 52.0 \%$, and $57.0 \% \mathrm{Cl}$ ) from Dr. Ehrenstorfer (Augsburg, Germany) and their mixtures (Table S1, Supporting Information) were used to establish a linear correlation between chlorine content and total response factor (see Fig. S1, Supporting Information (SI)) (Reth et al., 2005). Two individual injections were needed for each sample owing to the number of ions. Simultaneously comparing the retention time and chromatographic peak shapes of monitored [M $-\mathrm{Cl}]^{-}$ions could prove the identification of SCCP and MCCP congeners.

\section{2. $Q A / Q C$}

To ensure accurate identification and quantification of the target compounds, strict quality controls (SI) were employed. All the blank levels were negligible with $22.8 \pm 3.2 \mathrm{ng}$ for SCCPs and $17.7 \pm 3.8 \mathrm{ng}$ for MCCPs. The method detection limits (MDLs) were defined as the mean of all the blanks plus three times the standard deviation. The MDLs of SCCPs and MCCPs were estimated at 1.3 and $0.87 \mathrm{ng} / \mathrm{g}$ for sediments, 1.4 and $0.93 \mathrm{ng} / \mathrm{L}$ for filters, and 1.2 and $1.0 \mathrm{ng} / \mathrm{L}$ for the dissolved phase. The recoveries of ${ }^{13} \mathrm{C}_{10}$-trans-chlordane from all environmental samples were $76-107 \%$. All the samples were corrected by blanks, but not by surrogate recoveries. Detailed information about the analytical method is described in the supplementary information and in our latest study (Pan et al., 2018).

\subsection{Other parameters and statistical analyses}

Total organic carbon (TOC) and dissolved organic carbon (DOC) were determined for sediment and water samples, respectively. Other parameters, measured by a portable multi-parameter water quality meter (YSI Pro Plus, YSI Inc., USA), are presented in Table S2.

\section{Results and discussion}

Table S3 summarizes the concentrations of CPs in all the samples collected in the present study. The extract concentrate of the water phase sample at $\mathrm{X}_{14}$ was all split out during the pretreatment process, and no data were obtained. The calculated chlorine content for MCCPs in the dissolved phase and the sediments at $\mathrm{X}_{16}$ were far below the scope of the calibration curve. Even the smallest chlorine content could result in a large overestimation of the MCCP content. The sediment sample at site $\mathrm{X}_{13}$ exhibited similar values to those at $\mathrm{X}_{16}$ Therefore, the MCCP concentration data were not obtained from these samples. Except for the samples described above, the samples in this study fit well in the calibration curve. Based on the station locations, the sampling area was divided into two sections: river $\left(\mathrm{X}_{1}-\mathrm{X}_{23}\right)$ and Laizhou Bay $\left(\mathrm{X}_{24}-\mathrm{X}_{30}\right)$. The river section from site $\mathrm{X}_{10}$ to $\mathrm{X}_{23}$ was 


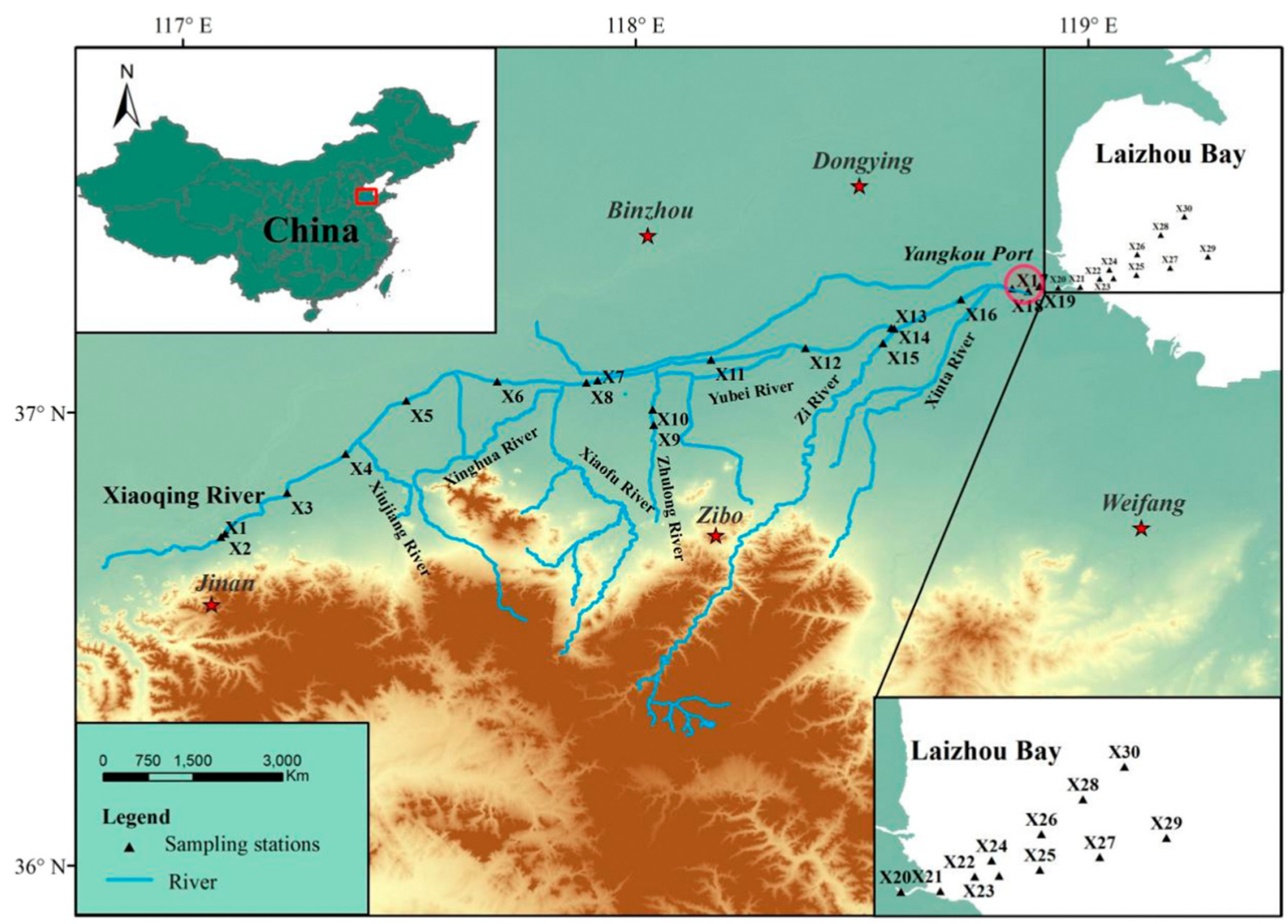

Fig. 1. Map of the sampling stations in the Xiaoqing River Basin.

influenced by the convergence of seawater and freshwater according to the salinity.

\subsection{CPs in sediments of the Xiaoqing River and Laizhou Bay}

The spatial distribution of the CPs is shown in Fig. 2a. The concentration amounts spanned three orders of magnitude. SCCP concentrations varied from 48 to $16,000 \mathrm{ng} / \mathrm{g} \mathrm{dw}$ along the river (sites $\mathrm{X}_{1}-\mathrm{X}_{23}$ ) with a mean value of $1300 \mathrm{ng} / \mathrm{g} \mathrm{dw}$ and ranged from 9.1 to $20 \mathrm{ng} / \mathrm{g} \mathrm{dw}$ in the Laizhou Bay ( sites $\mathrm{X}_{26}-\mathrm{X}_{30}$ ) with a mean of $13 \mathrm{ng} / \mathrm{g} \mathrm{dw}$. MCCPs concentrations ranged from 130 to $27,000 \mathrm{ng} / \mathrm{g} \mathrm{dw}$ in the river (mean value: $6020 \mathrm{ng} / \mathrm{g} \mathrm{dw}$ ) and varied from 2.4 to $9.0 \mathrm{ng} / \mathrm{g} \mathrm{dw}$ in the bay. MCCPs accounted for $52.0-98.4 \%$ of the summed CPs ( $\sum$ MCCPs $+\sum$ SCCPs) with a mean level of $79.6 \%$ in the river, and $17.6-44.0 \%$ (mean value: $29.6 \%$ ) in the bay. Three centralized peak concentrations appeared near $\mathrm{X}_{3}, \mathrm{X}_{10}-\mathrm{X}_{11}$, and $\mathrm{X}_{17}-\mathrm{X}_{19}$, which were located downstream of the cities of Jinan, Zibo, and in Yangkou Port (located in the estuary). Thus, the distinct influence of local discharge was clearly visible along the Xiaoqing River Basin.

The highest SCCP concentration occurred at site $\mathrm{X}_{10}$ (SCCP: $16,000 \mathrm{ng} /$ g dw; MCCP: 17,000 ng/g dw). In our previous studies, high concentrations of halogenated flame retardants (HFRs), decabromodiphenyl ethane (DBDPE), and per- and polyfluoroalkyl substances (PFASs) also occurred at $\mathrm{X}_{10}$ (Heydebreck et al., 2015; Zhen et al., 2018). Site $\mathrm{X}_{10}$ is located downstream of the city of Zibo and was sampled in the Zhulong River (a branch of the Xiaoqing River), which flows through several industrial parks in Zibo city. These contaminants with high concentrations at $\mathrm{X}_{10}$ indicated industrial discharge from Zibo city. The use of metal-working fluids during thermal processing in the machine processing industry has been recognized as a major source of SCCPs, contributing to $47.4 \%$ of the total emissions and $77.8 \%$ of water emissions in China (Jiang et al., 2017; Zhang et al., 2017). Zibo city is famous for machinery and for the processing industry (up to one hundred processing enterprises are located here), which might explain the high SCCP concentration at $\mathrm{X}_{10}$. The highest MCCP concentrations were found near Yangkou port $\left(\mathrm{X}_{18}\right.$ : $27,000 \mathrm{ng} / \mathrm{g} \mathrm{dw}, \mathrm{X}_{17}: 21,000 \mathrm{ng} / \mathrm{g} \mathrm{dw}$ ). Instead of industrial harbor, Yangkou port is a small-scale fishing and cargo port with a history of more than 100 years. Most of the moorings in Yangkou port are occupied by hundreds of traditional wooden fishing boats throughout the year. Several rubber tires hang on every boat just at the surface of the water. Waterproof and anticorrosive coatings are essential and brushed every year for repairment. CPs are commonly used as additives in the rubber industry, such as in marine coatings, rubber tires, and polyurethane materials (Tong et al., 2003; Wei et al., 2016). Many researchers have reported that compared to SCCPs, MCCPs are more prone to be adsorbed onto particles and settle in sediment instead of being transported by water over long distance (Chen et al., 2011; Ma et al., 2014; Pan et al., 2018; Zeng et al., 2013a). Thus, the mooring fishing boats might be a major contributor to MCCPs in the Xiaoqing River.

Both Pearson and Spearman correlations were performed between the MCCPs and SCCPs in the sediment samples. The results showed a significant correlation under Spearman analysis $(R=0.988, p<0.01)$ and a moderate correlation under Pearson analysis $(R=0.463$, $p<0.05$ ). This suggested the coexistence of SCCPs and MCCPs emissions at each site, but with different MCCP/SCCP ratios, suggesting a mixture of various pollution sources and/or different transport pathways. There was no significant correlation between SCCPs and TOC in the sediment samples of the Xiaoqing River, nor between MCCPs and TOC, which is mainly attributed to industrial discharge. Similar results were also reported for both the Liaohe River Basin and the Laizhou Bay areas (Gao et al., 2012; Pan et al., 2018).

In comparison, $\mathrm{CP}$ concentrations in the sediments of the Xiaoqing River were similar to those in the Pearl River Delta (SCCPs: 46.3-1540 ng/g, mean value $334 \mathrm{ng} / \mathrm{g}$; MCCPs: 102-6650 ng/g, mean value $1720 \mathrm{ng} / \mathrm{g}$ ), but higher than those in the Liaohe River Basin (39.8-480.3 ng/g). They were also higher than the concentrations in 

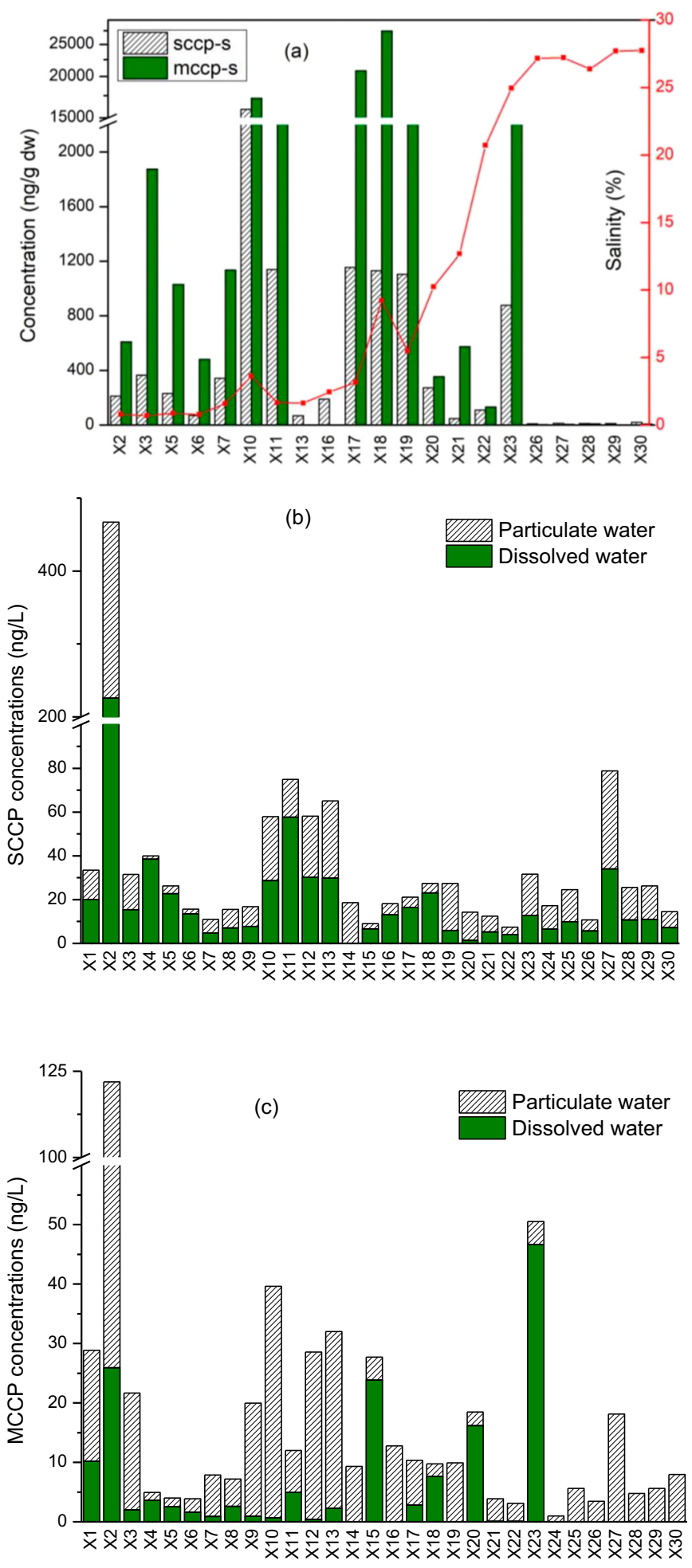

Fig. 2. Spatial distribution of SCCPs and MCCPs in the Xiaoqing River: (a) sediment; (b) SCCPs in dissolved and particulate phases; (c) MCCPs in dissolved and particulate phases.

the rivers of Japan, in the industrial area near Barcelona, Spain, in the rivers of the Czech Republic, and in all the reported coastal waters (Castells et al., 2008; Chen et al., 2011; Gao et al., 2012; Zeng et al., 2017). The highest SCCP concentration was found in rivers receiving industrial discharge in England (65,100 ng/g dw) (Nicholls et al., 2001), followed by that found at $\mathrm{X}_{10}$ in the present study. The SCCP concentration at $\mathrm{X}_{10}$ is the highest concentration reported in the last decade, much higher than that in a wastewater receiving lake in Beijing, China (8700 ng/g dw) (Zeng et al., 2012). MCCP concentrations found in the Xiaoqing River are much higher than those found in sewage sludge in Australia (Brandsma et al., 2017), and second only to those found in the Pearl River Delta (38,000 ng/g dw) (Chen et al., 2011). High CPconcentrations reflect the rapid industrial development, because the pollution level of CPs is reportedly directly proportional to industrialization (Zeng et al., 2017).

The compositional profiles of MCCPs varied little from the upstream to downstream and marine sediments (Fig. 3). The relative concentration of the congener groups declined with an increase in the carbon chain length, except for a few sites in the bay. $\mathrm{C}_{14}$ was dominant, with a percentage of $46.2 \pm 6.3 \%$. The dominant chlorinated groups were $\mathrm{Cl}_{7-8}$, followed by the $\mathrm{Cl}_{6}$ and $\mathrm{Cl}_{9}$ congener groups. For SCCPs, however, along with the sharp decrease in $\mathrm{CP}$ concentration from the river to the bay, the $\mathrm{CP}$ congener group patterns also presented obvious differences between the river section ( sites $\mathrm{X}_{1}-\mathrm{X}_{23}$ ) and the bay ( sites $\mathrm{X}_{26}-\mathrm{X}_{30}$ ), as shown in Fig. 4. In the river section, $C_{13}$ groups dominated in all the samples; they were slightly higher than in the $C_{11}$ and $C_{12}$ groups, while $C_{10}$ had the lowest percentage of them. On average, the $C_{10}-C_{13}$ congener groups contributed by $17.1 \pm 11.6 \%, 25.3 \pm 6.7 \%, 24.2 \pm 7.0 \%$, and $33.4 \pm 6.8 \%$, respectively. This is consistent with the industrial groups reported in our previous study and resembles those in the PRD and Shenzhen coastal sediments (Pan et al., 2018; Zeng et al., 2017). In the Laizhou Bay sediments of $\mathrm{X}_{26}-\mathrm{X}_{30}$, however, the $\mathrm{C}_{10}$ and $\mathrm{C}_{11}$ groups predominate, accounting for an average abundance of $50.7 \%$ and $32.8 \%$ respectively. Among the chlorine groups, $\mathrm{Cl}_{6-8}$ are the dominant congener groups in both the river section and the bay, accounting for $80 \%$ of the total. The $\mathrm{Cl}_{5-6}$ groups were elevated in the headwaters compared to their values in the marine sediments, while the $\mathrm{Cl}_{8-9}$ groups declined. Thus, for the sediments in the headwaters, the estuary, and in the bay, lighter congener groups with shorter carbon chains and lower chlorination tended to be transported for longer distances, while the heavier congeners were more likely to sink and deposit owing to larger $\mathrm{K}_{\mathrm{ow}}$ values. These findings have been stated in previous studies (Pan et al., 2018).

\subsection{CPs in waters of the Xiaoqing River and the bay}

$\mathrm{CP}$ concentrations ranged from n.d. to $470 \mathrm{ng} / \mathrm{L}$ for SCCPs (mean value: $43 \mathrm{ng} / \mathrm{L}$ ) and from n.d. to $120 \mathrm{ng} / \mathrm{L}$ for MCCPs (mean value: $28 \mathrm{ng} / \mathrm{L}$ ) in the water column (sum of the dissolved phase and particulate phase), as shown in Fig. 2b and c. SCCPs accounted for 95\% of CPs (sum of SCCPs and MCCPs) in the dissolved phase in the Xiaoqing River, ranging from n.d. to $230 \mathrm{ng} / \mathrm{L}$ with an average value of $23 \mathrm{ng} / \mathrm{L}$. The MCCPs ranged from n.d. to $47 \mathrm{ng} / \mathrm{L}$ with an average value of $5.9 \mathrm{ng} / \mathrm{L}$ in the dissolved phase. MCCPs existed mainly in the particulate phase and were closely spearman-correlated with SCCPs $(r=0.698, p<0.01)$. They were within the ranges of n.d. to $240 \mathrm{ng} / \mathrm{L}$ (mean value: $20 \mathrm{ng} / \mathrm{L}$ ) and n.d. to $96 \mathrm{ng} / \mathrm{L}$ (mean value: $13 \mathrm{ng} / \mathrm{L}$ ) for SCCPs and MCCPs in particulate phase, respectively. The highest concentration of SCCPs (470 ng/L) at $\mathrm{X}_{2}$ was even much higher than that found in the influent waters of the municipal sewage treatment plants in Beijing (184 ng/L) and Japan (279 ng/L) (Wei et al., 2016; Zeng et al., 2013b). X 2 was located along the main stream of the Xiaoqing River just below Jinan city; therefore, it might be influenced by urban emissions. However, other industrialized contaminants, such as perfluoroalkyl substances and halogenated flame retardants, were detected at relatively low concentrations, as reported in our previous studies (Heydebreck et al., 2015; Zhen et al., 2018). In addition, SCCP concentrations at $\mathrm{X}_{2}$ were more than 10 times higher than at $\mathrm{X}_{1}$, which was also located downstream of Jinan City and only about $460 \mathrm{~m}$ upstream of $\mathrm{X}_{2}$. When probing into the surroundings of sites $\mathrm{X}_{1}$ and $\mathrm{X}_{2}$, we found a drain outlet near site $X_{2}$ and determined that the discharged water was from a power plant used for refrigeration. SCCPs have been used as a polychlorinated biphenyl (PCB) substitute since PCBs were regulated in the 1970s (Fiedler, 2010), and have been widely used as an insulating 

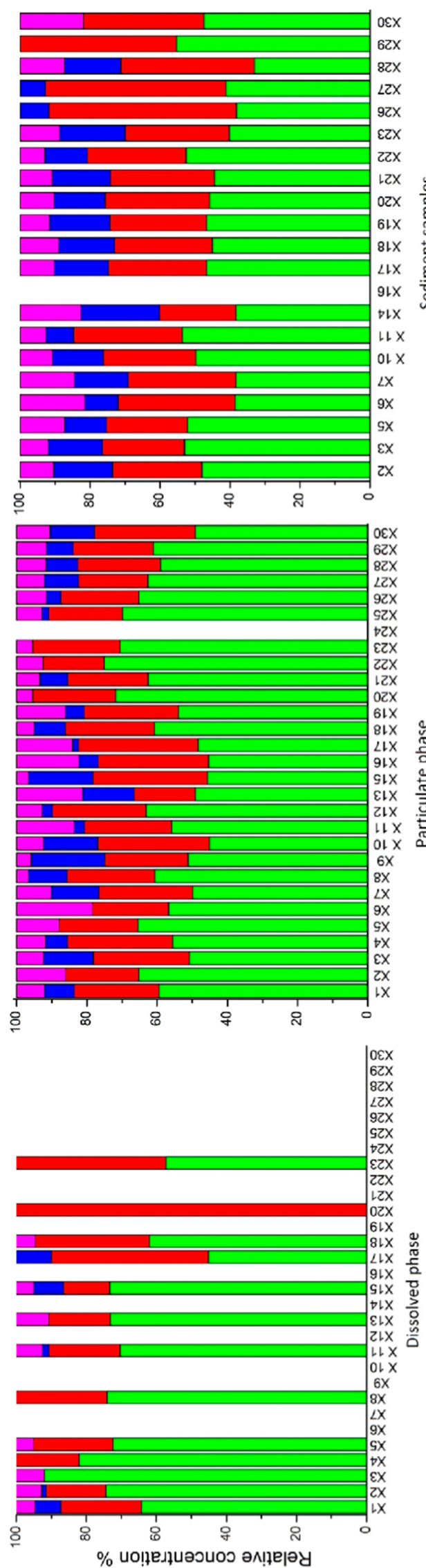

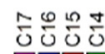

0000

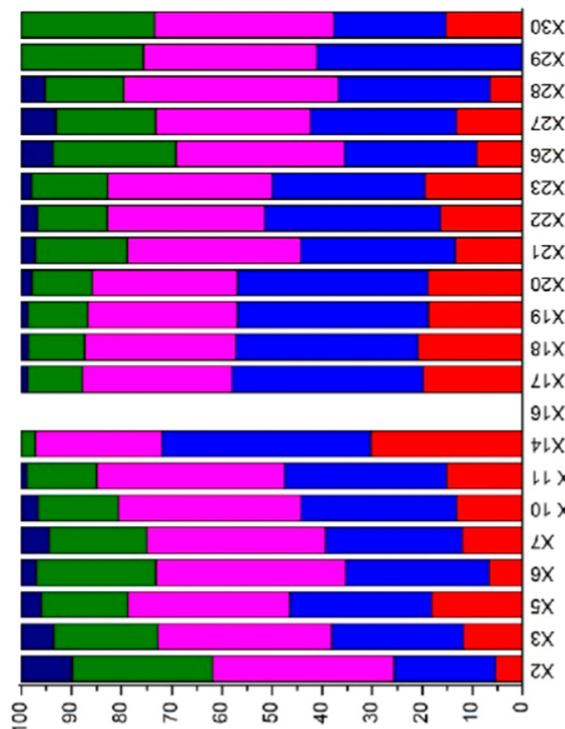

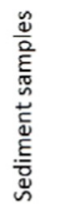
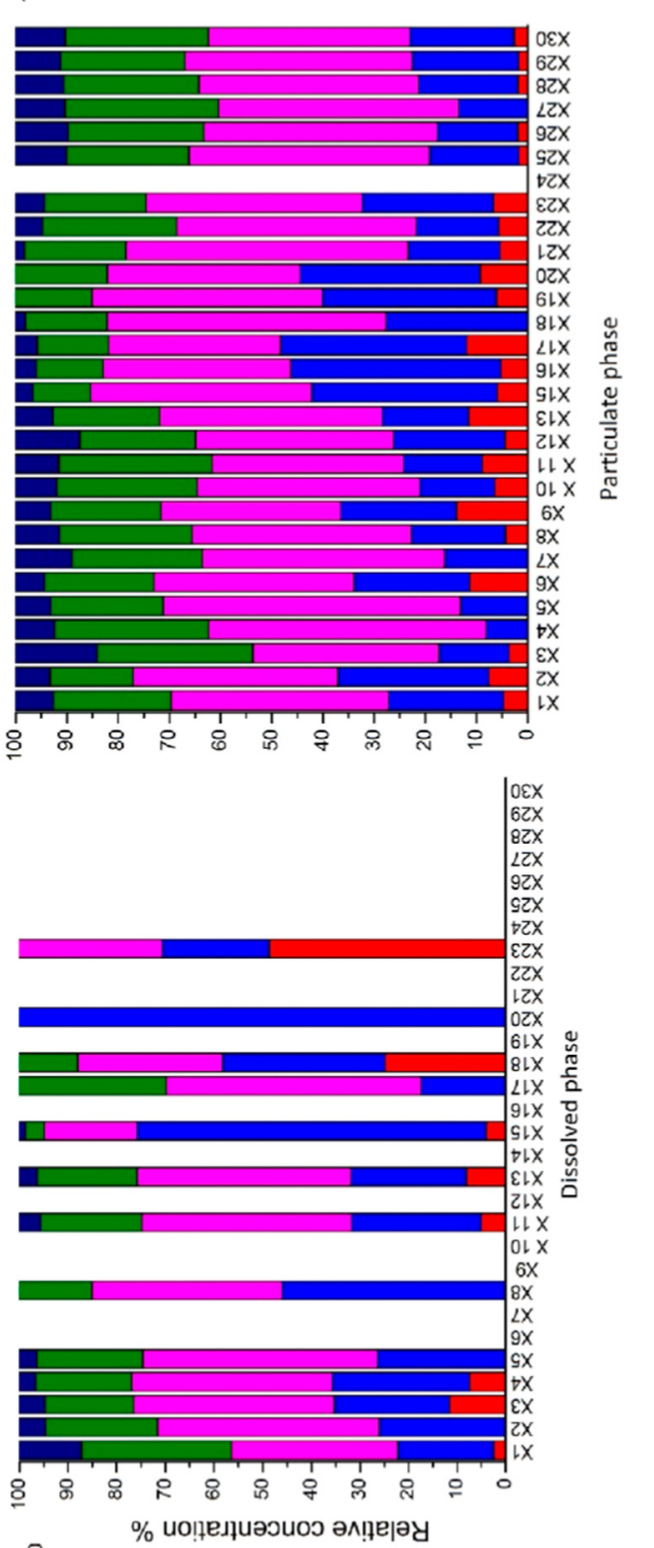

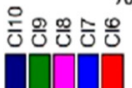



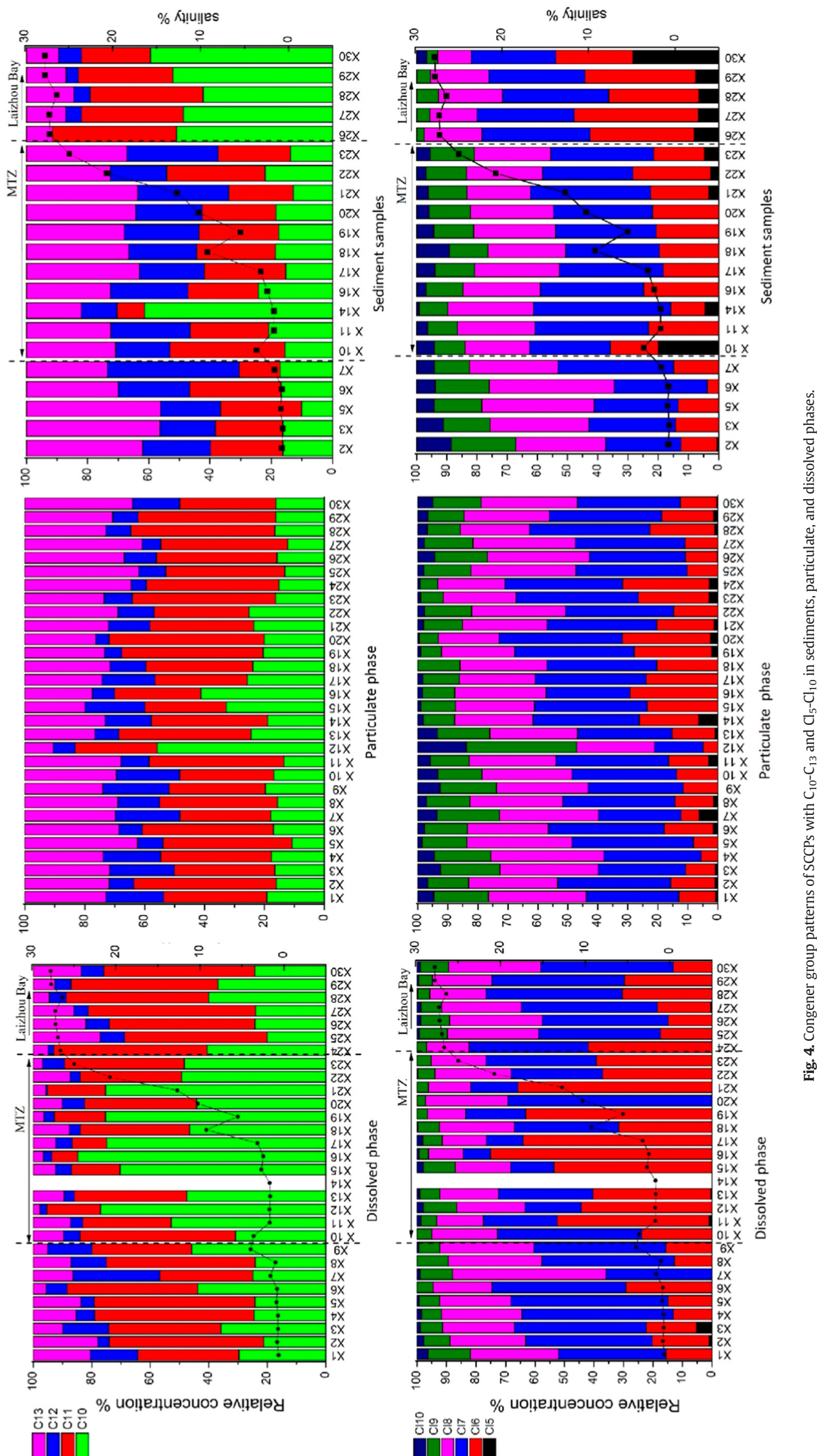
oil in transformers, capacitors, and other power equipment (Johnson et al., 1964). These may be the sources of SCCPs in the power plant.

The $C_{10}$ and $C_{11}$ groups were dominant congener groups in the dissolved phase, accounting for $81.6 \pm 9.2 \%$ of the total (Fig. 4). Among the chlorine groups, $\mathrm{Cl}_{6-8}$ accounted for $91.8 \pm 3.8 \%$ on average. For MCCPs, only $30 \%$ of $C_{16}$ or $C_{17}$ groups were detected in the dissolved phase. The $\mathrm{C}_{14}$ and $\mathrm{Cl}_{7-9}$ groups were predominant in almost all the sites (Fig. 3). In particulate samples, the dominant groups were $C_{11}$ and $\mathrm{C}_{13}$ groups, $\mathrm{Cl}_{7}$ and $\mathrm{Cl}_{8}$ groups (except X12), accounting for $38.5 \pm 7.1 \%\left(C_{11}\right), 28.6 \pm 5.9 \%\left(C_{13}\right), 35.1 \pm 5.2 \%\left(C_{7}\right)$, and $29.2 \pm$ $4.2 \%\left(\mathrm{Cl}_{8}\right)$ respectively. The congener group patterns of MCCP in particulate samples varied little across the different sites. $\mathrm{C}_{14}$ group was the predominant congener group, accounting for $87.6 \pm 4.6 \%$, followed by the $C_{15}$ group. $C_{16-17}$ accounted for less than $20 \%$ of the most samples. $\mathrm{Cl}_{7-9}$ groups were dominant, accounting for $58.7 \pm 9.8 \%$. However, owing to the method limitations, congener groups with more than 10 chlorine atoms could not be detected (Casà et al., 2019). Thus, the overestimation of the relative abundances of the congener groups detected in the present study might occur.

\subsection{Influence of environmental factors on the fate and transport of CPS along the estuary}

Relatively high CP concentrations were observed below Jinan $\left(\mathrm{X}_{2}-\mathrm{X}_{3}\right)$ and Zibo $\left(\mathrm{X}_{10}-\mathrm{X}_{11}\right)$ cities, indicating urban sources to the Xiaoqing River. Relatively high concentrations in water samples also occurred around the two city clusters. However, at sites $\mathrm{X}_{17}-\mathrm{X}_{19}$ that were sampled near Yangkou port however, $\mathrm{CP}$ concentrations in sediments were the highest, while those in water samples, especially in particulate samples, were lower than those in other sites. This contrast indicated a sedimentation of a historical input from a local source rather than of a current input, and was also due to the dilution by seawater in the water phase.

The estuarine turbidity maximum zone (ETM) is a typical characteristic of estuaries that determines water quality and influences the fate and transportation of pollutants. Fresh water and seawater converged at $\mathrm{X}_{10}$ 23, as shown by the salinity change curve, indicating that this section might be located within the ETM (Munoz et al., 2017). Studies have reported that the removal of contaminants from the water column may be enhanced at neap tides (low current velocities) as coarser particles settle on the bed sediment, and the 'salting-out' effects could be caused by a sharp increase in salinity (Munoz et al., 2017). Relatively higher concentrations of SCCPs were observed in the ETM of the Pearl River Estuary and the Liaohe Estuary than in the nearby upstream and downstream samples. These were related to a mixture of freshwater and seawater (Chen et al., 2011; Gao et al., 2012). In the present study, the mass distribution of lighter congener groups ( $\mathrm{C}_{10-11}$ and $\mathrm{Cl}_{6-7}$ groups) of SCCPs in the ETM declined in the order: dissolved phase $>$ particulate phase $>$ sediment, while the concentrations of heavier congeners $\left(\mathrm{C}_{13}\right.$ and $\mathrm{Cl}_{10}$ groups) increased. This variation within the ETM might be the result of both freshwater-seawater mixing and different physicochemical properties, such as LogKow values and solubilities. Long-chained and more chlorinated congeners with larger LogKow values might be more likely to be salted-out and thus be sequestrated in sediments, while lighter congener groups with relatively high water solubility might be more prone to be transported further with the water flow. This is probably also the main reason for the sharp decrease in $\mathrm{CP}$ concentrations and for the variation in congener groups from the river to the sea in sediments.

Other parameters, including dissolved organic carbon (DOC), salinity, $\mathrm{pH}$, oxidation-reduction potential (ORP), and vapor pressure, were simultaneously determined. It is interesting to note that CPs in the dissolved phase were significantly positively correlated with atmospheric pressure and that the coefficient for SCCPs was slightly higher than that for MCCPs. This result indicates the possible atmospheric input of the Xiaoqing River. Ma et al. (2018) reported the analysis of the net deposition in Liaodong Bay. They found that the diffusive inputs related to the concentrations and profiles of CPs in the dissolved phase in the water column were larger than the diffusive inputs from dry deposition related to CPs in the particulate phase CPs (Ma et al., 2018). Air-water gas exchange might be an important source of dissolved CPs in the waters of the Xiaoqing River and was enhanced by the tide's ebb and flow. Dissolved organic carbon (DOC) has been considered a key factor affecting the transportation and fate of persistent organic pollutants (POPs). However, in this study however, no significant correlation was observed between CPs and DOC, as well as between individual congener groups and the DOC in the water column, indicating that SCCPs mostly belonged to the freely dissolved category. This also further proved the role of the atmospheric input in the waters of the Xiaoqing River.

Correlations between SCCPs and MCCPs in the dissolved phase were greatly weakened and reduced $(r=0.39, p<0.05)$ compared to those in sediments and particles. The decline of the correlation coefficients from the sediment to the water phase reflected their different physicochemical properties and transfer-transformation behaviors. Compared with SCCPs, the congener patterns of MCCPs did not vary much from upstream to downstream and to the outer sea. There was only a slight decline in the $\mathrm{C}_{14}$-MCCP group in the following order: dissolved phase, particulate phase, and the sediment samples, which may be attributed to its strong hydrophobicity and weak solubility.

\subsection{Conclusion}

We have reported the occurrence of SCCPs and MCCPs in the Xiaoqing River basin. Both water and sediment samples were analyzed to explore the transport behaviors and fate from the river to the Laizhou Bay. The results revealed that cities are an important pollution source of CPs pollution in the river environment. In the Xiaoqing River, relatively high concentrations occurred when the river passed by Jinan and Zibo cities. The highest SCCP concentration at $\mathrm{X}_{10}$ is the highest concentration reported in the last decade, even higher than that in a wastewater-receiving lake in Beijing. MCCP concentrations exhibited the second highest values ever recorded, second only to those found in the Pearl River Delta. The mass distribution analysis indicated that long-chained and more chlorinated congeners with larger LogKow values ( $\mathrm{C}_{13}$ and $\mathrm{Cl}_{10}$ groups) were more likely to be salted-out and be sequestrated within the ETM, while the lighter congener groups $\left(C_{10-11}\right.$ and $\mathrm{Cl}_{6-7}$ groups), having relatively high water solubility, were prone to be transported further away with the water flow. This is probably an important influencing factor affecting the congener pattern differences from the river to the Laizhou Bay.

\section{CRediT authorship contribution statement}

Xiaohui Pan: Investigation, Methodology, Data curation, Software, Writing - original draft. Xiaomei Zhen: Investigation, Data curation, Visualization, Software, Validation. Chongguo Tian: Methodology, Validation, Writing - review \& editing. Jianhui Tang: Conceptualization, Supervision, Data curation, Funding acquisition, Resources, Project administration, Writing - review \& editing.

\section{Declaration of competing interest}

The authors declare that they have no known competing financial interests or personal relationships that could have appeared to influence the work reported in this paper.

\section{Acknowledgement}

This study was supported by the National Natural Science Foundation of China (Grant Nos. U1806207, 41103074 and 41773138), by the seed project of Yantai Institute of Coastal Zone Research, Chinese Academy of Sciences (No. YIC Y855011024) and the Key deployment project of Centre for Ocean Mega-Science, Chinese Academy of Sciences (COMS2019J08). 


\section{Appendix A. Supplementary data}

Supplementary data to this article can be found online at https://doi. org/10.1016/j.scitotenv.2020.141769.

\section{References}

Bayen, S., Obbard, J.P., Thomas, G.O., 2006. Chlorinated paraffins: a review of analysis and environmental occurrence. Environ. Int. 32, 915-929.

Brandsma, S.H., van Mourik, L., O’Brien, J.W., Eaglesham, G., Leonards, P.E.G., de Boer, J., et al., 2017. Medium-chain chlorinated paraffins (CPs) dominate in Australian sewage sludge. Environ. Sci. Technol. 51, 3364-3372.

Brits, M., de Boer, J., Rohwer, E.R., De Vos, J., Weiss, J.M., Brandsma, S.H., 2020. Short-, medium-, and long-chain chlorinated paraffins in South African indoor dust and cat hair. Chemosphere 238, 124643.

Casà, M.V., van Mourik, L.M., Weijs, L., Mueller, J., Nash, S.B., 2019. First detection of shortchain chlorinated paraffins (SCCPs) in humpback whales (Megaptera novaeangliae) foraging in Antarctic waters. Environ. Pollut. 250, 953-959.

Castells, P., Parera, J., Santos, F.J., Galceran, M.T., 2008. Occurrence of polychlorinated naphthalenes, polychlorinated biphenyls and short-chain chlorinated paraffins in marine sediments from Barcelona (Spain). Chemosphere 70, 1552-1562.

Chen, M.-Y., Luo, X.-J., Zhang, X.-L., He, M.-J., Chen, S.-J., Mai, B.-X., 2011. Chlorinated paraffins in sediments from the Pearl River Delta, South China: spatial and temporal distributions and implication for processes. Environ. Sci. Technol. 45, 9936-9943.

Du, X., Yuan, B., Zhou, Y., Benskin, J.P., Qiu, Y., Yin, G., et al., 2018. Short-, medium- and long-chain chlorinated paraffins in wildlife from paddy fields in the Yangtze River Delta. Environ. Sci. Technol. 52, 1072-1080.

Fiedler, H., 2010. Short-chain chlorinated paraffins: production, use and international regulations. In: Boer, J. (Ed.), Chlorinated Paraffins. Springer Berlin Heidelberg, Berlin, Heidelberg, pp. 1-40.

Gao, Y., Zhang, H., Su, F., Tian, Y., Chen, J., 2012. Environmental occurrence and distribution of short chain chlorinated paraffins in sediments and soils from the Liaohe River Basin, P. R. China. Environ. Sci. Technol. 46, 3771-3778.

Glüge, J., Schinkel, L., Hungerbühler, K., Cariou, R., Bogdal, C., 2018. Environmental risks of medium-chain chlorinated paraffins (MCCPs): a review. Environ. Sci. Technol. 52, 6743-6760.

Heydebreck, F., Tang, J., Xie, Z., Ebinghaus, R., 2015. Alternative and legacy perfluoroalkyl substances: differences between European and Chinese river/estuary systems. Environ. Sci. Technol. 49, 8386-8395.

Huang, H., Gao, L., Zheng, M., Li, J., Zhang, L., Wu, Y., et al., 2018. Dietary exposure to shortand medium-chain chlorinated paraffins in meat and meat products from 20 provinces of China. Environ. Pollut. 233, 439-445.

Iozza, S., Schmid, P., Oehme, M., 2009. Development of a comprehensive analytical method for the determination of chlorinated paraffins in spruce needles applied in passive air sampling. Environ. Pollut. 157, 3218-3224.

Jiang, W., Huang, T., Mao, X., Wang, L., Zhao, Y., Jia, C., et al., 2017. Gridded emission inventory of short-chain chlorinated paraffins and its validation in China. Environ. Pollut. 220, 132-141.

Johnson, G.W., Quensen, I.I.I.J.F., Chiarenzelli, J.R., Hamilton, M.C., 1964. 10 polychlorinated biphenyls. In: Morrison, R.D., Murphy, B.L. (Eds.), Environmental Forensics. Academic Press, Burlington, pp. 187-225.

Ma, X., Zhang, H., Wang, Z., Yao, Z., Chen, J., Chen, J., 2014. Bioaccumulation and trophic transfer of short chain chlorinated paraffins in a marine food web from Liaodong Bay, North China. Environ. Sci. Technol. 48, 5964-5971.

Ma, X., Wang, Y., Gao, W., Wang, Y., Wang, Z., Yao, Z., et al., 2018. Air-seawater gas exchange and dry deposition of chlorinated paraffins in a typical inner sea (Liaodong Bay), North China. Environ. Sci. Technol. 52, 7729-7735.
Munoz, G., Budzinski, H., Labadie, P., 2017. Influence of environmental factors on the fate of legacy and emerging per- and polyfluoroalkyl substances along the salinity/turbidity gradient of a macrotidal estuary. Environ. Sci. Technol. 51, 12347-12357.

Nicholls, C.R., Allchin, C.R., Law, R.J., 2001. Levels of short and medium chain length polychlorinated n-alkanes in environmental samples from selected industrial areas in England and Wales. Environ. Pollut. 114, 415-430.

Pan, X., Tang, J., Li, J., Zhong, G., Chen, Y., Zhang, G., 2011. Polybrominated diphenyl ethers (PBDEs) in the riverine and marine sediments of the Laizhou Bay area, North China. J. Environ. Monit. 13, 886-893.

Pan, X., Tang, J., Tian, C., Li, J., Zhang, G., 2018. Short- and medium-chain chlorinated paraffins in sediments from the Laizhou Bay area, North China: implications for transportation from rivers to marine environment. Environ. Pollut. 243, 1460-1468.

Reth, M., Oehme, M., 2004. Limitations of low resolution mass spectrometry in the electron capture negative ionization mode for the analysis of short- and medium-chain chlorinated paraffins. Anal. Bioanal. Chem. 378, 1741-1747.

Reth, M., Zencak, Z., Oehme, M., 2005. New quantification procedure for the analysis of chlorinated paraffins using electron capture negative ionization mass spectrometry. J. Chromatogr. A 1081, 225-231.

Sprengel, J., Wieselmann, S., Kröpfl, A., Vetter, W., 2019. High amounts of chlorinated paraffins in oil-based vitamin E dietary supplements on the German market. Environ. Int. 128, 438-445.

Tong, L. Li, J., Sun, Y., 2003. The present situation and development of chlorinated rubber and paint industry. In chinese. Paint and Coatings industry 33, 39-60.

UNEP, 2016. Short-chain chlorinated paraffins (SCCPs): risk management evaluation. UNEP/POPS/POPRC.12/11/Add.3. Twelfth meeting of the Persistent Organic Pollutants Review Committee. Rome, Italy. http://chm.pops.int/Default.aspx?tabid=5171.

Wei, G.-L., Liang, X.-L., Li, D.-Q., Zhuo, M.-N., Zhang, S.-Y., Huang, Q.-X., et al., 2016. Occurrence, fate and ecological risk of chlorinated paraffins in Asia: a review. Environ. Int (92-93), 373-387.

Zeng, L., Wang, T., Ruan, T., Liu, Q., Wang, Y., Jiang, G., 2012. Levels and distribution patterns of short chain chlorinated paraffins in sewage sludge of wastewater treatment plants in China. Environ. Pollut. 160, 88-94.

Zeng, L., Chen, R., Zhao, Z., Wang, T., Gao, Y., Li, A., et al., 2013a. Spatial distributions and deposition chronology of short chain chlorinated paraffins in marine sediments across the Chinese Bohai and Yellow Seas. Environ. Sci. Technol. 47, 11449-11456.

Zeng L, Li, H., Wang T, Gao, Y, Xiao, K., Du, Y., et al., 2013b. Behavior, fate, and mass loading of short chain chlorinated paraffins in an advanced municipal sewage treatment plant. Environ. Sci. Technol. 47, 732-740.

Zeng, L., Lam, J.C.W., Chen, H., Du, B., Leung, K.M.Y., Lam, P.K.S., 2017. Tracking dietary sources of short- and medium-chain chlorinated paraffins in marine mammals through a subtropical marine food web. Environ. Sci. Technol. 51, 9543-9552.

Zhang, R., Zhang, G., Zheng, Q., Tang, J., Chen, Y., Xu, W., et al., 2012. Occurrence and risks of antibiotics in the Laizhou Bay, China: impacts of river discharge. Ecotoxicol. Environ. Saf. 80, 208-215.

Zhang, B., Zhao, B., Xu, C., Zhang, J., 2017. Emission inventory and provincial distribution of short-chain chlorinated paraffins in China. Sci. Total Environ. (581-582), 582-588.

Zhao, Z., Tang, J., Xie, Z., Chen, Y., Pan, X., Zhong, G., et al., 2013. Perfluoroalkyl acids (PFAAs) in riverine and coastal sediments of Laizhou Bay, North China. Sci. Total Environ. 447, 415-423.

Zhen, X., Tang, J., Liu, L., Wang, X., Li, Y., Xie, Z., 2018. From headwaters to estuary: distribution and fate of halogenated flame retardants (HFRs) in a river basin near the largest HFR manufacturing base in China. Sci. Total Environ. 621, 1370-1377.

Zhong, G., Tang, J., Zhao, Z., Pan, X., Chen, Y., Li, J., et al., 2011. Organochlorine pesticides in sediments of Laizhou Bay and its adjacent rivers, North China. Mar. Pollut. Bull. 62, 2543-2547. 